\title{
Dinâmica populacional canina: potenciais efeitos de campanhas de esterilização
}

\author{
Marcos Amaku, ${ }^{1}$ Ricardo Augusto Dias ${ }^{1}$ e Fernando Ferreira ${ }^{1}$
}

Como citar

Amaku M, Dias RA, Ferreira F. Dinâmica populacional canina: potenciais efeitos de campanhas de esterilização. Rev Panam Salud Publica. 2009;25(4):300-4.

RESUMO Objetivo. Analisar, por meio de modelos matemáticos, os potenciais impactos das campanhas de esterilização na redução da densidade populacional de cães domiciliados.

Métodos. Foram elaborados modelos matemáticos para simular a dinâmica populacional de cães domiciliados e projetar os resultados de estratégias de controle para diferentes taxas de esterilização.

Resultados. Mesmo para altas taxas de esterilização (por exemplo, 0,80 ano-1), seriam necessários cerca de 5 anos de campanhas para possibilitar uma redução de $20 \%$ na densidade. No entanto, outras fontes de crescimento populacional, como a importação de cães de outras áreas, poderiam reduzir a efetividade dos programas de esterilização.

Conclusões. A efetividade de um programa depende não apenas da taxa de esterilização, mas também da taxa de crescimento populacional. As campanhas de esterilização podem potencialmente reduzir a densidade populacional, embora a redução nem sempre possa ser notada de imediato.

Palavras-chave Dinâmica populacional, cães, controle da população, esterilização reprodutiva, simulação por computador, Brasil.

O número crescente de cães abandonados é uma preocupação para as autoridades de saúde pública em vários países. Alguns agravos à saúde, como transmissão de algumas zoonoses e acidentes por mordedura, podem ser associados às populações de cães (1). A esterilização de cães domiciliados, a eutanásia de cães abandonados e as campanhas educativas são exemplos de me-

\footnotetext{
Universidade de São Paulo, Faculdade de Medicina Veterinária e Zootecnia. Enviar correspondência a Marcos Amaku no seguinte endereço: Avenida Prof. Dr. Orlando Marques de Paiva 87, CEP 05508-270, São Paulo, SP, Brasil. Fone: +55-113091.1388; fax: +55-11-3091.7928; e-mail: amaku@ usp.br, amaku@vps.fmvz.usp.br
}

didas tomadas na tentativa de resolver esse problema. É difícil, no entanto, estimar a efetividade dessas estratégias para reduzir a população de cães errantes, particularmente em curtos períodos de tempo. Essa questão não é simples, entre outros porque depende de fatores culturais, sociais, econômicos e comportamentais relacionados à posse responsável de animais. Por exemplo, há programas (2) que recomendam a esterilização de cães domiciliados; no entanto, alguns proprietários são contrários à esterilização de seus animais, o que reduz a eficácia desse tipo de medida.

Vários estudos já foram desenvolvidos sobre a dinâmica populacional canina, com foco principalmente em cães domiciliados, na densidade populacional canina, na razão cão:homem e em aspectos de distribuição etária dos animais (3-8). Mesmo assim, pouca atenção tem sido dada à avaliação dos efeitos de programas de esterilização e eutanásia. Dessa forma, o objetivo deste estudo foi estimar a efetividade e os efeitos da esterilização na redução da densidade populacional canina, utilizando modelos matemáticos que simulam a dinâmica populacional.

\section{MATERIAIS E MÉTODOS}

O modelo utilizado parte das seguintes pressuposições: o método de con- 
trole (esterilização) é implementado continuamente (a taxas anuais constantes); a esterilização dura a vida toda; a população está sujeita à densidadedependência (ou seja, a velocidade de crescimento da população depende da densidade populacional, diminuindo à medida que a densidade se aproxima da capacidade de suporte do ambiente); todos os jovens nascem férteis; e há um efeito de densidade-dependência sobre a natalidade, no qual a capacidade de suporte é dada pela razão cão:homem, uma vez que o tamanho da população canina depende do tamanho da população humana.

O modelo matemático determinístico desenvolvido para simular a dinâmica da população canina domiciliada é descrito a seguir.

\section{Crescimento populacional}

Para o presente estudo, adaptamos o modelo de Barlow et al. (9) para simular os efeitos de esterilização e sacrifício em populações animais selvagens.

Considerando que $N(t)$ é a densidade populacional animal em função do tempo $t$, assumimos que a população apresenta um crescimento logístico densidadedependente, dado pela seguinte equação:

$$
\frac{d N(t)}{d t}=N[f(N)-g(N)]
$$

Nessa primeira equação (equação 1), $f(N)$ e $g(N)$ são funções relacionadas à natalidade (ou recrutamento) e à mortalidade, respectivamente. Pressupõe-se que $f(N)-g(N)=r(1-N / K)$. Pressupõe-se também que, para uma população canina domiciliada, há um recrutamento densidade-dependente que pode ser modelado pela equação 2:

$$
\begin{aligned}
& f(N)=a-r \frac{N}{K} \\
& g(N)=b
\end{aligned}
$$

Na equação 2, $K$ é a capacidade de suporte, $a$ é a taxa de recrutamento (composta pela taxa de natalidade bruta $a_{0} \mathrm{e}$ uma taxa de recrutamento adicional $\alpha$ relacionada a outras fontes de crescimento populacional canino, como, por exemplo, importação de cães de outras regiões), $b$ é a taxa de mortalidade e $r$ (que é igual $a-b$ ) é a taxa de crescimento. Na equação 2, podemos ver que, quando $N=K$, a função de natalidade $f(N)$ e a função de mortalidade $g(N)$ são iguais.

\section{Controle por esterilização}

Caughley et al. (10) argumentam que um conhecimento da estrutura social e do sistema de acasalamento é desejável antes que o controle populacional pela supressão da fertilidade das fêmeas seja aplicado. No presente trabalho, levaremos em conta o cenário 1 descrito por Barlow et al. (9), que se aplica tanto ao acasalamento poligâmico, com esterilização de machos ou fêmeas ou apenas de fêmeas, quanto ao acasalamento monogâmico, com esterilização de animais de apenas um dos sexos. Concentraremos a nossa atenção na esterilização de fêmeas porque a proporção de fêmeas que não se reproduzem corresponde aproximadamente à probabilidade de esterilização (9).

Seja $Q$ a proporção de fêmeas que não se reproduzem; por conseguinte, $1-Q$ é a fração da população que é capaz de se reproduzir. Incluindo o efeito da esterilização, temos uma redução na função de natalidade, dada por $f(N)(1-Q)$. Assim, a equação 1 pode ser reescrita como

$$
\left.\frac{d N(t)}{d t}=N[f(N)(1-Q)-g(N)] \quad \text { (equação } 3\right) .
$$

Sejam $S, v$ e $\sigma$, respectivamente, a densidade de fêmeas que não se reproduzem, a proporção de fêmeas na população e a taxa de esterilização. Dividindo-se a densidade de fêmeas que não se reproduzem $S$ pela densidade total de fêmeas $v N$, obtemos a proporção de fêmeas que não se reproduzem, conforme a equação 4 :

$$
Q=\frac{S}{v N} \text {. }
$$

Para estabelecer uma relação entre a proporção de fêmeas esterilizadas, $Q$, e a taxa de esterilização $\sigma$, devemos levar em conta a equação 5:

$$
\frac{d S}{d t}=-g(N) S+\sigma(v N-S) .
$$

$\mathrm{Na}$ equação 5 , o primeiro termo do lado direito, $-g(N) S$, está relacionado à mortalidade de fêmeas, e o segundo, $\sigma(v N-S)$, é a densidade de novas fêmeas esterilizadas por unidade de tempo, uma vez que $v N-S$ é a densidade total de fêmeas não-esterilizadas.

Diferenciando $Q$ (equação 4) em relação ao tempo $t$, e levando em conta as equações 3 e 5, obtém-se a equação 6 :

$$
\frac{d Q}{d t}=(1-Q)(\sigma-Q f(N)) \text {. }
$$

As equações 3 e 6 foram usadas para estimar os efeitos do controle por esterilização. Para longos períodos de tempo, as soluções das equações 3 e 6 tendem a se estabilizar, e os pontos de equilíbrio $\left(N^{*}\right.$ e $\left.Q^{*}\right)$ são dados por

$$
\begin{aligned}
& N^{*}=0 \text { e } Q^{*}=1, \text { para } \sigma>a, \\
& N^{*}=0 \text { e } Q^{*}=\frac{\sigma}{a}, \text { para } r<\sigma<a(\text { equação } 7) \\
& N^{*}=K\left(1-\frac{\sigma}{r}\right) \text { e } Q^{*}=\frac{\sigma}{\sigma+b}, \text { para } \sigma<r .
\end{aligned}
$$

\section{Estimativas de parâmetros}

Para estimar os efeitos da esterilização em cães domiciliados, utilizamos taxas vitais para a população canina de General Pico, na Argentina, em 1990 (4) e também para uma população canina hipotética. Foram considerados, portanto, os seguintes parâmetros: taxa de natalidade $a_{0}$ de 0,366 ano $^{-1}$ para General Pico e de $0,70 \mathrm{ano}^{-1}$ para a população hipotética; e taxa de mortalidade $b$ de 0,157 ano $^{-1}$ para General Pico (mortalidade neonatal, já que não havia estimativa disponível para a taxa de mortalidade bruta) e de 0,167 ano $^{-1}$ para a população hipotética. Para estimar a taxa de natalidade na população canina hipotética, consideramos um modelo exponencial simples em que cada fêmea seria capaz de gerar dois filhotes por ano. Para estimar a taxa de mortalidade, assumimos uma mortalidade do tipo II (11), em que a taxa é constante e equivalente ao inverso da expectativa de vida. $\mathrm{O}$ valor adotado para a expectativa de vida foi 6 anos.

Por simplicidade, neste trabalho, assumimos uma capacidade de suporte de 1000 cães por $\mathrm{km}^{2}$, semelhante à densidade observada na Cidade de São Paulo em 2002 (estimativa de 987,74 cães por $\mathrm{km}^{2}$ ) (12). As equações diferenciais foram solucionadas numericamente através do método de Runge-Kutta de quarta ordem, com a auxílio do programa MathCad 2001.

\section{RESULTADOS}

A figura 1 mostra a densidade canina domiciliada relativa, dada pela densidade de cães dividida pela capacidade de suporte $K$, em função do tempo, para diferentes taxas de esterilização, considerando-se a população canina como fechada, ou seja, sem importação de animais de outras áreas $(\alpha=0)$. Para a população canina de General Pico (fi- 
FIGURA 1. Densidade canina e densidade canina relativa ( $N / K$ : densidade/capacidade de suporte) em função do tempo para diferentes taxas de esterilização de cães

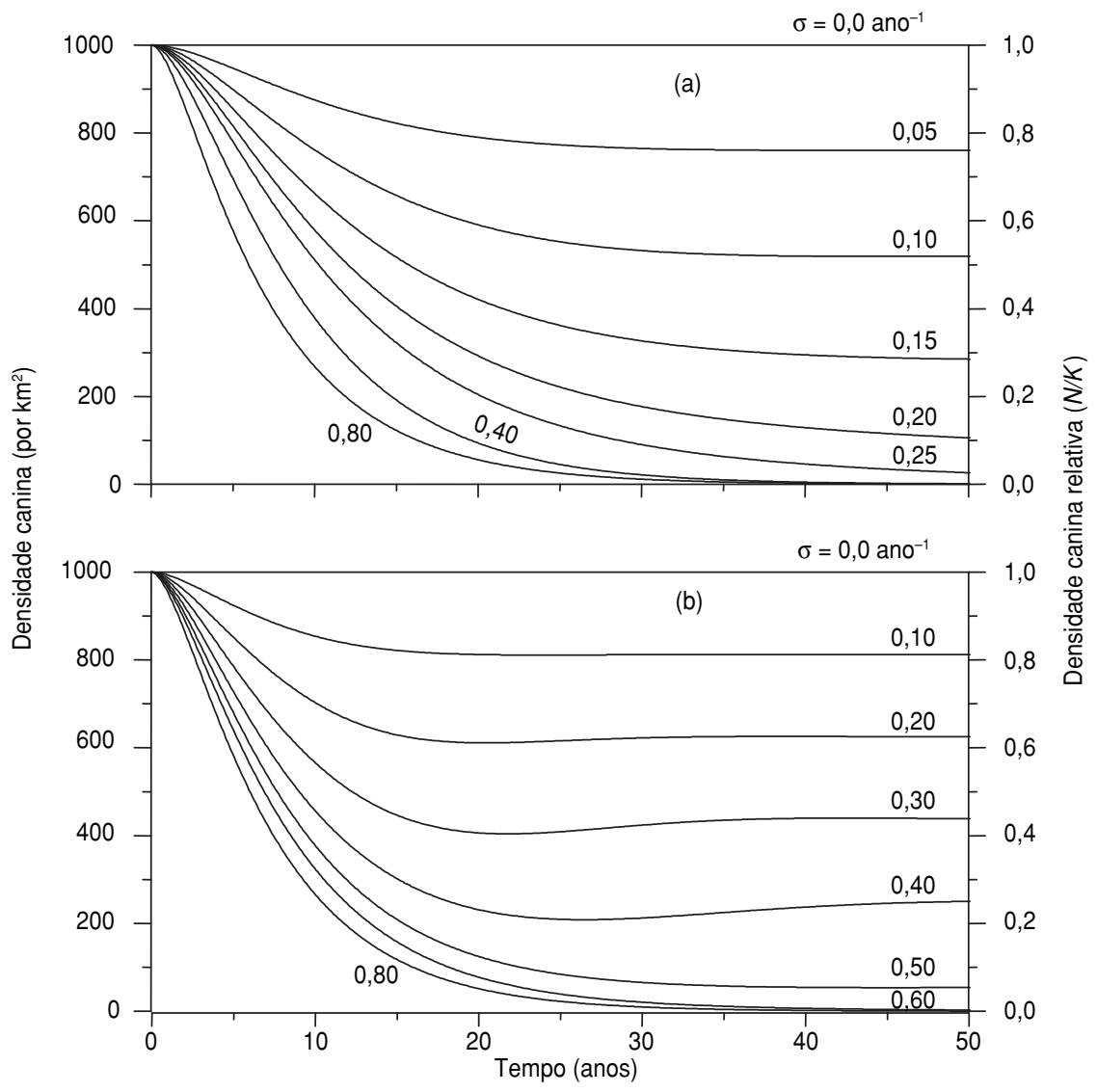

a) População canina de General Pico, Argentina; b) população canina hipotética.

gura 1a), pode-se notar que a esterilização a taxas acima da taxa de crescimento natural $\left(0,209\right.$ ano $\left.^{-1}\right)$ produz uma redução maior do que $90 \%$ na população canina a longo prazo. Uma taxa de 0,40 $\mathrm{ano}^{-1}$ é suficiente para eliminar a população canina após 40 anos. Para atingir uma redução de $20 \%$ na densidade populacional, para uma taxa de esterilização de 0,40 $\mathrm{ano}^{-1}$, seriam necessários cerca de 5 anos. Para taxas inferiores a 0,40 ano $^{-1}$, são necessários mais do que 5 anos para atingir uma redução de $20 \%$.

Para a população hipotética (figura 1b), os aspectos gerais são semelhantes aos observados na figura 1a. No entanto, como a taxa de crescimento é de 0,533 ano $^{-1}$, mais elevada que a taxa da população canina de General Pico, seriam necessárias, para atingir níveis de redução semelhantes aos observados na figura 1a, taxas de esterilização mais elevadas. Por exemplo, para uma redução maior do que $90 \%$ na população canina, seria necessária uma taxa de esterilização maior do que 0,50 ano $^{-1}$. A figura 1 mos- tra ainda que o tempo necessário para atingir o estado de equilíbrio varia de acordo com a taxa de esterilização, sendo em geral superior a 20 anos.

Analisando os pontos de equilíbrio (equação 7), notamos que, quando $\sigma>r$, a população se extingue após um longo período de tempo. Quando $\sigma<r, N$ estabiliza em $N^{*}=K\left(1-\frac{\sigma}{r}\right)$.

A figura 2 mostra a densidade populacional canina em função do tempo para uma taxa de esterilização fixa de 0,40 ano $^{-1}$ e diferentes valores da taxa de recrutamento $\alpha$, usando os parâmetros para a população canina de General Pico. Podemos observar que, para uma dada taxa de esterilização, as densidades de equilíbrio são maiores para valores maiores da taxa de recrutamento adicional $\alpha$.

\section{DISCUSSÃO}

As campanhas de esterilização têm sido adotadas em várias localidades como estratégias para controlar a popu- lação canina (13). No entanto, devido à ausência de estudos que analisem quantitativamente o impacto de tais campanhas, não é possível, no momento, comparar os resultados de nosso modelo com resultados de campo. Por um lado, em algumas cidades, a esterilização não obteve os resultados desejados devido a uma taxa de esterilização baixa (13). Por outro lado, em Jaipur, Índia, a porcentagem de fêmeas caninas esterilizadas chegou a $70 \%$ (14).

As simulações (figura 1) mostraram que a esterilização aplicada continuamente ao longo do tempo é capaz de reduzir a densidade populacional canina se não houver importação de animais de outras áreas. No entanto, mesmo para altas taxas de esterilização (por exemplo, 0,80 $\mathrm{ano}^{-1}$ ), uma redução de $20 \%$ na densidade populacional seria notada apenas após cerca de 5 anos de campanhas de esterilização. Por conseguinte, o modelo indica que o impacto desse tipo de programa de rotina não seria notado imediatamente.

O impacto de uma dada taxa de esterilização na densidade canina depende da taxa de crescimento da população. Esse resultado é também esperado teoricamente para a densidade de equilíbrio (equação 7), dado que, para $\sigma>r$, a população se extingue após um período de tempo. Por outro lado, para $\sigma<r$, a população se torna estável em $N^{*}=$ $K\left(1-\frac{\sigma}{r}\right)$. Na ausência de esterilização $(\sigma=0)$, a população se estabiliza na capacidade de suporte.

Observamos, na figura 2, que, para uma dada taxa de esterilização, as densidades de equilíbrio são mais altas para valores mais elevados da taxa de recrutamento adicional $\alpha$. Embora seja um resultado direto, isso pode explicar por que, em algumas comunidades, os programas de esterilização parecem não ter reduzido de modo significativo a população canina.

Concentramos a nossa atenção em taxas críticas e aspectos temporais, tais como o tempo necessário para atingir o equilíbrio. Esses aspectos são importantes para comparar os efeitos a longo prazo das estratégias baseadas em diferentes taxas de controle. Complementarmente aos aspectos temporais, os aspectos etários são também importantes. De fato, algumas limitações da abordagem do presente trabalho, tais como considerar taxas médias para natalidade e mortalidade, são relacionados à 
FIGURA 2. Densidade canina e densidade relativa ( $N / K$ : densidade/capacidade de suporte) em função do tempo para uma taxa de esterilização fixa de 0,40 ano $^{-1}$ e diferentes valores de taxa de recrutamento adicional $\alpha\left(\right.$ ano $\left.^{-1}\right)$, usando os parâmetros para a população canina de General Pico, Argentina

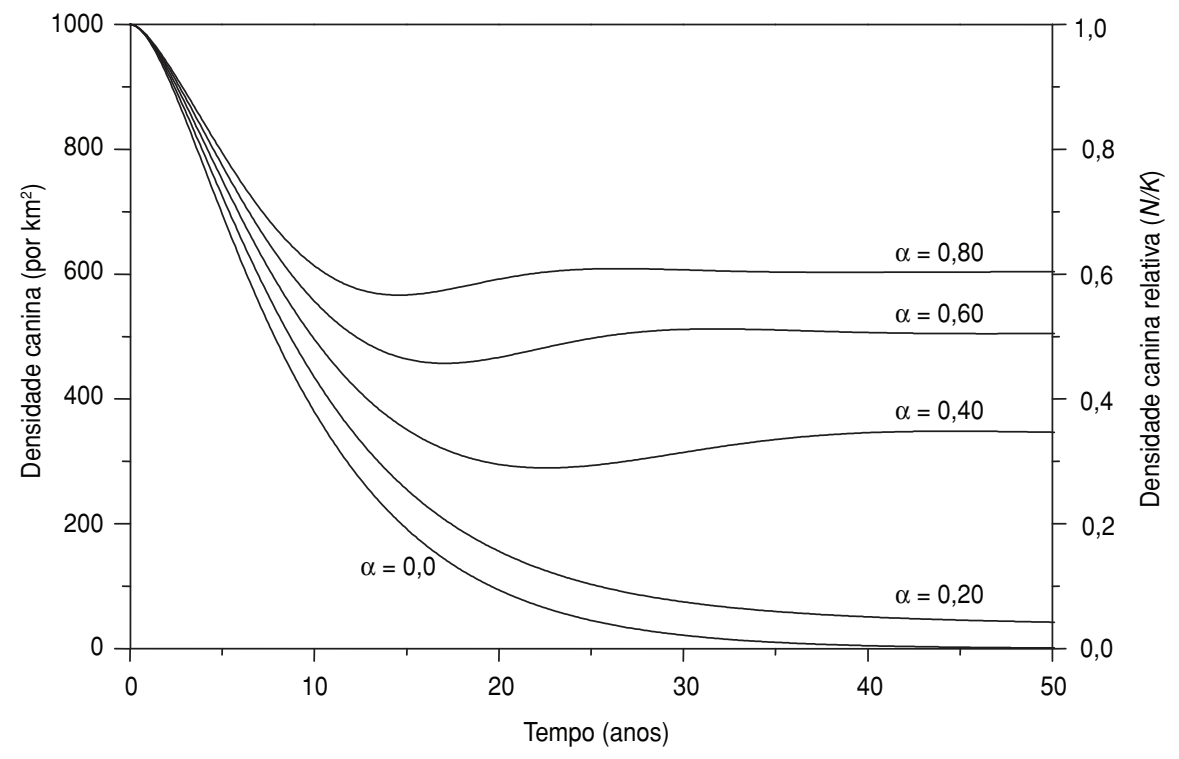

dependência etária. Embora essas limitações possam afetar as propriedades dos estados estacionários atingidos, os modelos apresentados levam em conta os principais aspectos que permitiriam decidir, por meio de comparações relativas, qual estratégia de controle seria mais apropriada. Os assuntos relacionados à dependência etária, como idades ótimas para esterilização, serão abordados em estudos futuros.

Embora o problema do abandono de cães não tenha sido abordado diretamente no presente trabalho, poder-se-ia esperar que campanhas de esterilização levassem à redução da taxa de abandono.
No entanto, a redução das taxas de abandono depende da posse responsável de animais; assim, não é simples modelar como a esterilização afetaria o abandono. Embora seja possível organizar campanhas educacionais para promover a posse responsável, sua efetividade dependerá da resposta da população, o que, por sua vez, pode depender de aspectos culturais.

Um potencial impacto das campanhas de esterilização para a saúde pública, relacionado à redução da densidade populacional e da taxa de abandono, seria reduzir, indiretamente, o número de agressões de cães a pessoas e, por conseguinte, os custos do tratamento médico às vítimas de agressão com mordedura. Por exemplo, a incidência média anual de mordeduras por cães errantes foi de 95,64 por 100000 habitantes no período de 2003 a 2006 em Belgrado, Sérvia (15).

Embora seja uma questão polêmica, a esterilização de cães errantes foi adotada em alguns locais (13). Um modelo semelhante ao apresentado neste trabalho pode ser aplicado para estimar os efeitos desses programas, sendo que o nosso grupo desenvolveu uma análise dos potenciais efeitos de estratégias de controle na dinâmica populacional de cães errantes (16).

Finalmente, é importante salientar que os resultados das simulações que apresentamos foram baseados em um conjunto particular de parâmetros e suposições do modelo. Essas suposições, assim como os parâmetros estimados, podem ser modificados para modelar a dinâmica populacional canina e os efeitos de estratégias de esterilização em uma comunidade específica. Nesse contexto, seria interessante a realização de estudos complementares, que permitam avaliar tanto a relação custo-benefício dessas estratégias quanto a acurácia das estimativas do modelo. Estudos longitudinais de campo - atualmente, por exemplo, o nosso grupo está desenvolvendo um estudo em que a população de cães de uma localidade é acompanhada periodicamente - poderão também fornecer subsídios para que sejam superadas as limitações de tempo observadas na modelagem.

Agradecimentos. Este trabalho contou com o apoio do Conselho Nacional de Desenvolvimento Científico e Tecnológico (CNPq), projeto 476531/2003-9.

\section{REFERÊNCIAS}

1. Beck A. The ecology of stray dogs. West Lafayette: Purdue University Press; 1973.

2. Viaro O, Paranhos NT. Para viver de bem com os bichos (manual do educador). São Paulo: Centro de Controle de Zoonoses; 2002.

3. Dias RA, Garcia RC, Silva DF, Amaku M, Ferreira Neto JS, Ferreira F. Estimativa de populações canina e felina domiciliadas em zona urbana do Estado de São Paulo. Rev Saude Publica. 2004;38(4):565-70.

4. Larrieu E, Alvarez T, Cavagion L, Herrasti A. Dinamica de la poblacion canina de General Pico, Argentina en el periodo 1986/1990. Vet Arg. 1992;9(88):536-41.
5. Marx MB, Furcolow ML. What is the dog population? A review of survs in the United States. Arch Environ Health. 1969;19(2):217-9.

6. Nassar R, Mosier JE. Canine population dynamics: a study of the Manhattan, Kansas, canine population. Am J Vet Res. 1980;41(11): 1798-803.

7. Nunes CM, Martines DA, Fikaris S, Queiróz LH. Avaliação da população canina da zona urbana do município de Araçatuba, São Paulo, SP, Brasil. Rev Saude Publica. 1997; 31(3):308-9.

8. Santamaria A, Passannanti S, Di Franza D. Censimento dei cani randagi in un quartiere di Napoli. Acta Med Vet. 1990;36:201-13.
9. Barlow ND, Kean JM, Briggs CJ. Modelling the relative efficacy of culling and sterilisation for controlling populations. Wildl Res. 1997;24(2):129-41.

10. Caughley G, Pech R, Grice D. Effect of fertility control on a population's productivity. Wildl Res. 1992;19(6):623-7.

11. Anderson RM, May RM. Infectious diseases of humans: dynamics and control. Oxford: Oxford University; 1991.

12. Grisi-Filho JHH, Amaku M, Dias RA, Netto HM, Paranhos NT, Mendes MCNC, Ferreira Neto JS. Uso de sistemas de informação geográfica em campanhas de vacinação contra a raiva. Rev Saude Publica. 2008;42(6):1005-11. 
13. Clifton M. Sterilization and vaccination: $70 \%$ or flunk. Disponível em: http://www.best friends.org/nomorehomelesspets/pdf/thres hold.pdf. Acessado em fevereiro de 2009.

14. Conservation Research. A report to WSPA by Conservation Research Ltd on work carried out at the Help in Suffering animal shelter in Jaipur, Rajasthan. Cambridge: Conserva- tion Research; 2007. Disponível em: www. conservationresearch.co.uk/straydog/HIS report07.doc. Acessado em fevereiro de 2009.

15. Marijana V, Dordević M, Brana RD, Ljiljana J, Mirilović M. Bites to humans caused by stray and owned dogs in Belgrade. Acta Vet-Beogr. 2008;58(5-6):563-71.
16. Amaku M, Dias RA, Ferreira F. Modeling stray dog population dynamics and control Math Popul Stud. 2009;no prelo.

Manuscrito recebido em 28 de janeiro de 2008. Aceito em versão revisada em 15 de abril de 2008

ABSTRACT Objective. To analyze, through mathematical modeling, the potential ability of sterilization campaigns to reduce the population density of pet dogs.

\section{Canine population dynamics: the potential effect of sterilization campaigns}

Methods. Mathematical models were constructed to simulate the canine population dynamics and project the results of control strategies based on several sterilization rates.

Results. Even at high sterilization rates (for example, 0.80 year $^{-1}$ ), it would take approximately 5 years to reduce density by $20 \%$. Even so, other sources of population growth, such as the importing of dogs from other geographic areas, could outweigh the effects of a sterilization program.

Conclusions. A program's effectiveness is contingent upon not only on the sterilization rate, but also the rate of population growth. Sterilization campaigns may potentially reduce population density, but this reduction may not be immediately evident.

Key words Population dynamics, dogs; population control; sterilization, reproductive; computer simulation, Brazil.

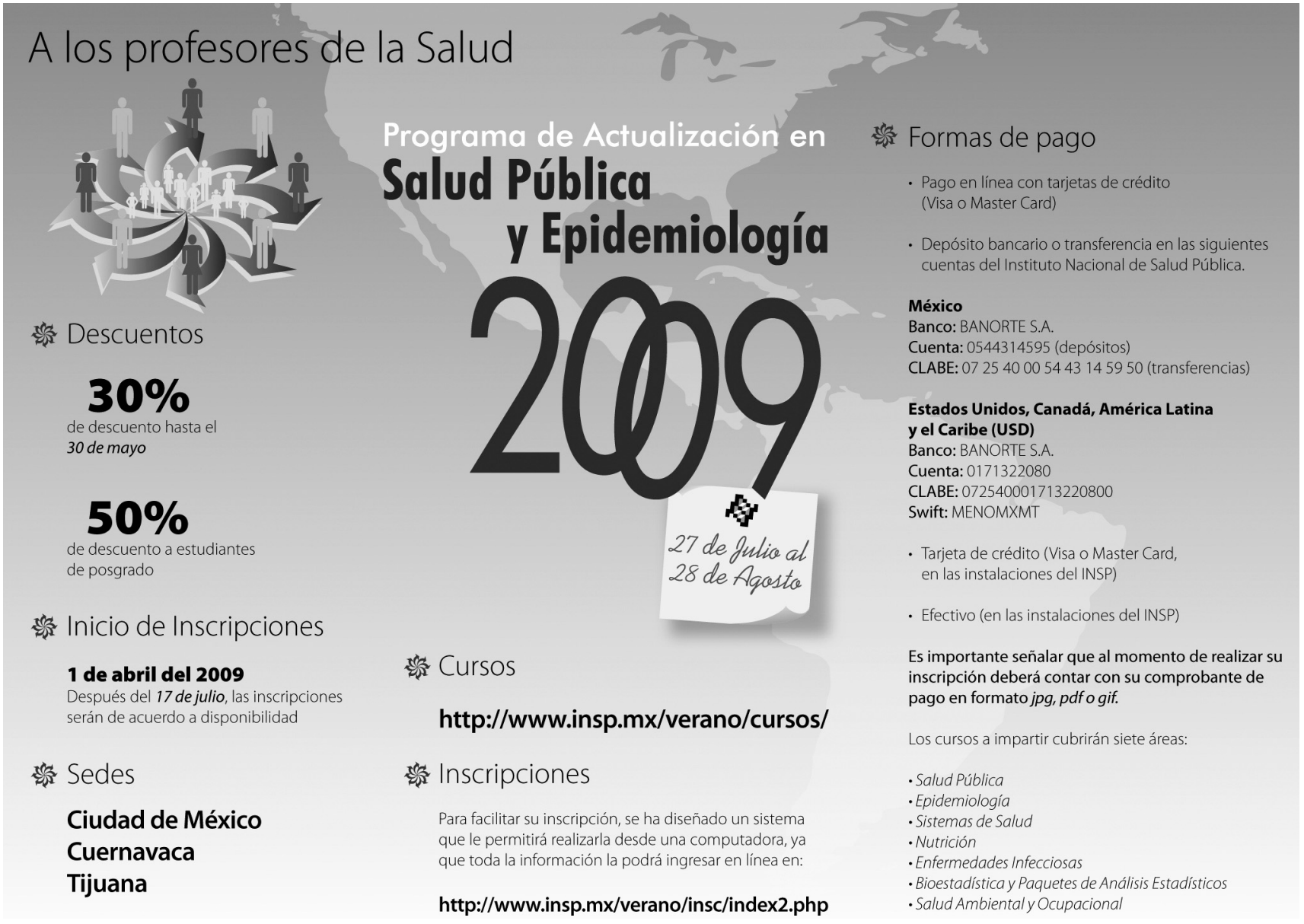

